\title{
La carbonización de residuos biomásicos: una exploración con perspectivas emocionantes
}

\author{
The carbonization of biomass waste: \\ an exploration with exciting prospects
}

Jaime Quesada Kimzey'

Fecha de recepción: 20 de enero del 2012

Fecha de aprobación: 19 de marzo del 2012

Quesada, J. La carbonización de residuos biomásicos: una exploración con perspectivas emocionantes. Tecnología en Marcha. Vol. 25, № 5. Pág |4-2|.

Este artículo cuenta con el aval de la Vicerrectoría de Investigación y Extensión del 


\section{Resumen}

Sedaunaidea general sobreel tema delacarbonización de residuos biomásicos, y se describen brevemente dos proyectos en esta área de investigación que en el primer semestre del 2012 se desarrollan en el Tecnológico de Costa Rica (TEC). Ambos proyectos están enfocados en la carbonización de residuos biomásicos del beneficiado del café y se realizan en colaboración con Coopetarrazú. El que dio inicio en el 20 I I está dedicado a la carbonización de material seco, y abordará tanto el uso energético como el uso agrícola del biocarbón. El que dio inicio en el 2012 está enfocado en la carbonización hidrotérmica de biomasa presente en las aguamieles.

\section{Palabras clave}

Carbonización, biomasa, residuos, energía renovable, biocarbón.

\begin{abstract}
This paper offers a general view of the subject of carbonization of waste biomass. Just as well, it briefly describes two related projects currently under execution at the TEC. Both projects are focused on carbonization of waste biomass from the industrial processing of coffee, in a joint effort with Coopetarrazú. The project initiated in 2011 is dedicated to carbonization of dried wastes and will explore energetic as well as agricultural use of the charcoal. The one initiating in 2012 focuses on the hydrothermal carbonization of biomass in waste slurries.
\end{abstract}

\section{Key words}

Carbonization, biomass, waste, renewable energy, biochar.

\section{Introducción}

Nuestra época es una en la que se descubren día a día valores nuevos e inesperados en antiguos conocimientos, tecnologías y materiales, heredados de generaciones desaparecidas. La carbonización de biomasa ha sido una actividad humana al menos desde que se descubrió el valor del carbón así obtenido (biocarbón, para distinguirlo del carbón mineral) como combustible. La era del hierro fue posible gracias al biocarbón: sin él, no se podía alcanzar la temperatura de fusión del hierro, ni se podía sin carbón obtener hierro del mineral que lo contenía.

Pero no está claro cuándo se descubrió el valor del biocarbón, como mejorador casi milagroso de suelos. Este descubrimiento está evidenciado en antiquísimas "terras pretas" halladas en nuestro continente principalmente, algunas en sitios que no se sabía que habían sido habitados alguna vez (I, 2, 3). El biocarbón evidentemente ha acompañado a la humanidad más tiempo y con más implicaciones de las que suponíamos hasta hace poco.

El biocarbón actualmente reviste gran interés desde la perspectiva de las energías renovables. A partir de la consciencia de la importancia de sustituir los combustibles fósiles por otras fuentes de energía, se posiciona la biomasa como fuente de energía, de bioenergía. Materiales tan diversos como la leña, residuos agroindustriales, etanol, aceites, aceites pirolíticos, y (bio) carbones han pasado bajo la lupa.

Los materiales transformados, como aceites, etanol y biocarbón, aventajan a la biomasa de la que se originan en la concentración de la energía por unidad de masa o volumen, que es cerca del doble o mayor. Pero los usos que se exploran en la actualidad no se reducen al energético, sino que incluyen el potencial del biocarbón en el mejoramiento de suelos, la fabricación de carbones nanoestructurados y carbones activados para usos especializados como la purificación de aguas o la catálisis industrial, y la funcionalización química superficial del biocarbón para usos especiales. Todo a partir de biomasa.

\section{La biomasa}

Para obtener biocarbón, hay que empezar por la biomasa. Biomasa es cualquier material proveniente de seres vivos, pero evidentemente se refiere principalmente a los materiales más abundantes. También suele usarse el término para referirse a la masa de la materia viva en un sistema como el marino. 


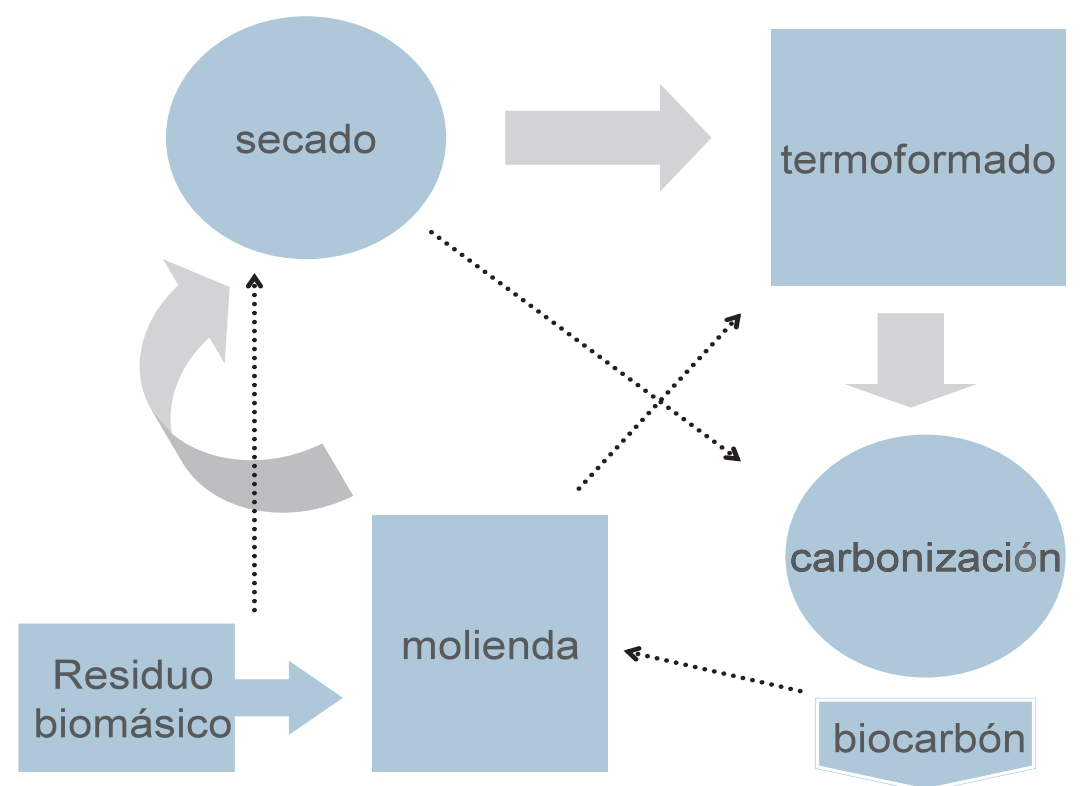

Figura I. Esquema funcional del proceso de carbonización en seco, aplicado al equipo del proyecto. Las flechas punteadas indican alternativas aplicables para materiales particulados, como la broza y la cascarilla.

Se puede obtener biocarbón de casi cualquier biomasa, pero es particularmente apropiada la de origen vegetal, sobre todo la constituida por materiales lignocelulósicos, que son el constituyente predominante de la vegetación terrestre, después del agua. La biomasa se puede extraer de una variedad de fuentes, como por ejemplo, a partir de bosques primarios y secundarios, a partir de cultivos destinados a generar biomasa, o a partir de residuos de cultivos agrícolas y forestales.

La biomasa se puede utilizar como fuente de bioenergía, mediante su combustión. Se devuelve al aire el carbono que la vegetación extrajo de él utilizando energía solar, en el proceso que llamamos fotosíntesis, y se libera la energía almacenada, que es de origen solar. Las tecnologías existentes actualmente para este propósito requieren de secar la biomasa, sea como un paso previo o durante el proceso, para poder quemarla. En el uso energético de la biomasa, el costo del transporte es determinante para su rentabilidad. Cuanto menos densa sea una biomasa, mayor será la tasa de costo de transporte / valor energético.

Para explicar esto con un ejemplo, los residuos de un cultivo, cuando están secos se pueden quemar para generar muchísima energía. El valor económico de esa energía, sin embargo, se perdería con solo tener que transportar esos residuos por más de unos $10 \mathrm{~km}$ sin haberlos secado, en algunos casos. Si se transportaran secos por más de unos $30 \mathrm{~km}$, se harían poco rentables, principalmente porque ocupan mucho espacio. Así que es necesario someter el material a procesos que concentren la energía que contiene, en menor volumen y menor masa, de modo que el costo de su transporte no le quite su rentablilidad a esa energía. La carbonización es uno de tales procesos de concentración.

\section{El secado de la biomasa}

En la actualidad, para obtener energía de biomasa, primero hay que secar la biomasa, sea durante el proceso o como paso previo. La modalidad de secado es determinante para la rentabilidad de la bioenergía contenida en residuos agrícolas. Sobre el secado hay que dejar claro que, para no socavar la rentabilidad de la bioenergía en cuestión, debe lograrse mediante calores residuales de procesos existentes, o bien mediante energía solar. Para obtener biocarbón con las tecnologías actuales, también hay que secar la biomasa. Al cierre de este artículo se aborda nuevamente este tema al mencionar una nueva tecnología de carbonización que podría permitirnos evadir la cuestión del secado de la biomasa, tecnología que trataremos de implementar y desarrollar en el TEC próximamente. 


\section{La preferencia por los residuos}

En todo el mundo, el manejo de los residuos y desechos representa un costo para las empresas. En los países en que hay regulaciones efectivas, este manejo puede ser bastante costoso para las empresas que generan los desechos, mientras que en países con regulaciones más laxas, las empresas pueden evadir sus responsabilidades y trasladar esos costos, multiplicados, a la sociedad civil en forma de daño ambiental. Entonces el menor costo de producción, con el subsecuente menor precio del producto, se traduce en un costo ambiental que afecta a toda la población en su salud y calidad de vida: se "socializan". La decisión de dónde se paga, si en el producto con dinero, o en el ambiente con calidad de vida y salud, usualmente no la toma la población.

El dilema descrito en el párrafo anterior, afortunadamente, es innecesario. Los residuos agrícolas y agroindustriales pueden ser, en lugar de un problema costoso, fuente de empleo y bienestar para todas las personas. Los residuos generados anualmente por procesos agrícolas y agroindustriales en Costa Rica contienen una cantidad de energía

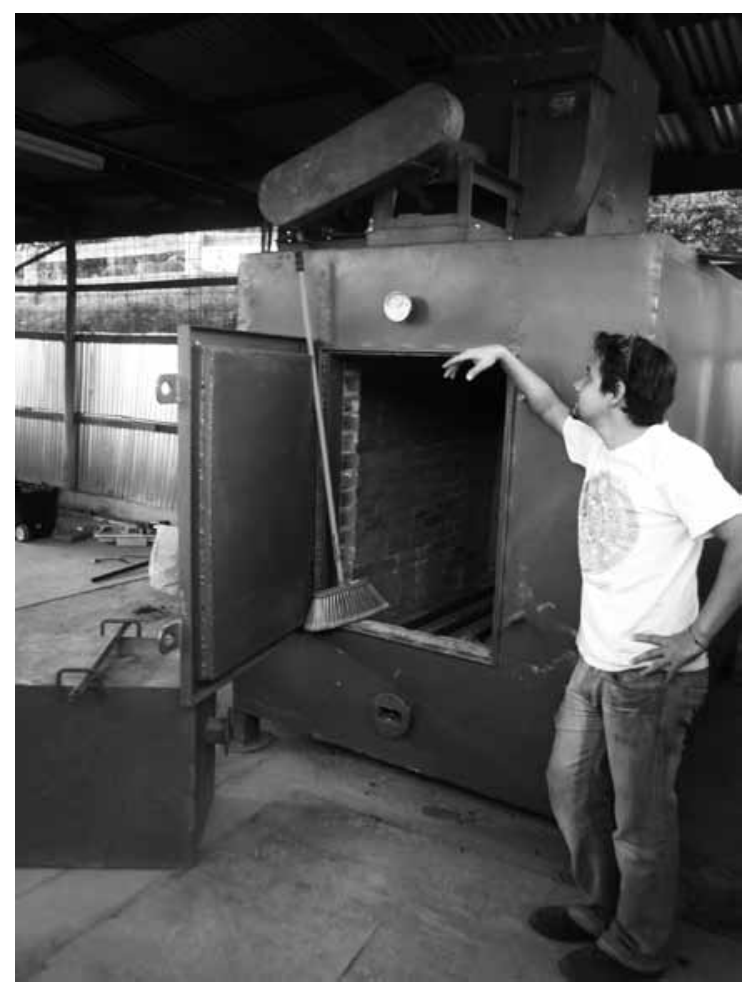

Horno de carbonización, con capacidad de $3 \mathrm{~m}^{3}$. Puede procesar más de 500 kg por tanda. mayor que la importada anualmente en forma de combustibles fósiles (4), con un costo cercano a los US\$2 000 millones (5). En lugar de dejar que esos residuos simplemente se pudran, ocasionando en muchos casos toda una variedad de problemas ambientales y costos de manejo, se pueden obtener empleos y ganancias de ellos mediante su aprovechamiento energético. Con ello, se podrían evitar los costos y daños ambientales, y generar empleo y bienestar, junto con las consecuencias positivas para la economía, derivadas de la sustitución de importaciones de combustibles fósiles.

La sustitución de combustibles fósiles tiene una variedad de ventajas ambientales por sí misma. La primera y más obvia es evitar la inyección de carbono fósil al ciclo biogeoquímico global, evitando el empeoramiento del efecto invernadero. Pero además, los combustibles fósiles son, en muchos casos, mucho más contaminantes que su contraparte biomásica. Hay un detalle importante, que suele ser considerado una desventaja, pero en realidad es una ventaja de la bioenergía a partir de residuos: La conversión de residuos biomásicos en formas utilizables para la obtención de energía, que sean ambientalmente razonables, puede ser más compleja que la contraparte de las fuentes fósiles. Esta mayor complejidad se traduce en un número mayor de pasos intermedios, con mayor labor humana involucrada.

El significado de esta mayor complejidad puede ser, desde una perspectiva económica, una mayor distribución de las ganancias entre un número mayor de familias. Así, lo que podría calificarse como menor rentabilidad, significaría un beneficio que se vería en la sociedad costarricense en forma de más fuentes de empleo y de ingresos para familias de estratos medios y bajos. Finalmente, la evitación de daños ambientales representa una ventaja significativa, e incluye una amplia gama, desde olores, humos tóxicos, moscas y otras plagas, hasta lixiviados que contaminan aguas superficiales y subterráneas.

En resumen, no aprovechar la bioenergía contenida en residuos agrícolas y agroindustriales es un enorme desperdicio, y además tiene un alto costo. 


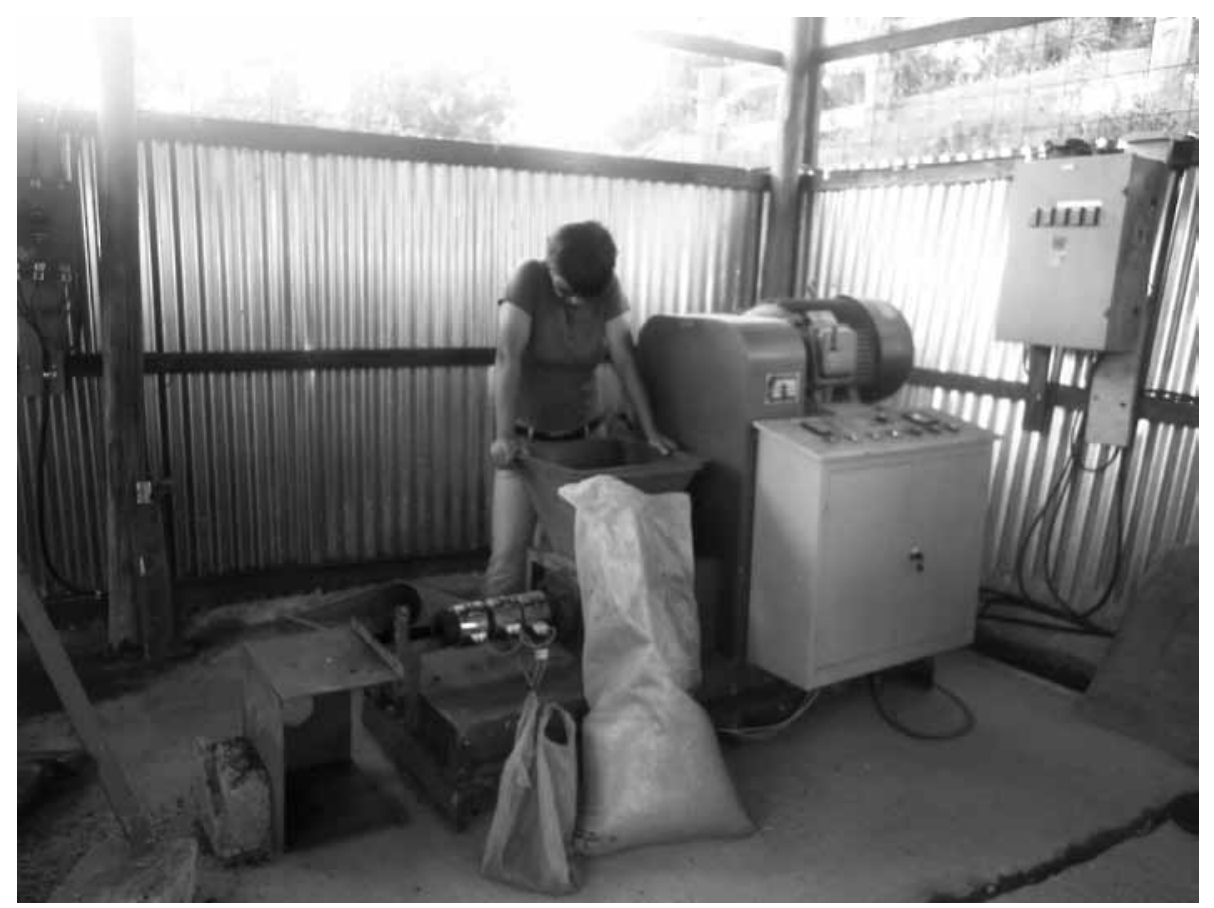

Briqueteador de biomasa por extrusión en caliente. Puede producir cerca de $100 \mathrm{~kg}$ de briquetas por hora.

\section{¿Por qué carbonizar?}

El carbón de biomasa, o biocarbón, tiene a su más conocido representante en el carbón de leña. Sin embargo, casi cualquier material de origen vegetal puede convertirse en biocarbón, con un proceso similar al aplicado a la leña. La densidad energética del biocarbón es cerca del doble de la que tiene una biomasa densa, como la leña. El propósito principal de carbonizar una biomasa con propósitos energéticos es el de reducir los costos de manejo y transporte. Se pierde energía en el proceso, pero se hace más factible la utilización de la que queda. La carbonización tiene un beneficio adicional, que es evitar la emisión de humo, dado que el carbón produce esencialmente $\mathrm{CO}_{2}$ y $\mathrm{CO}$ al quemarse.

El biocarbón tiene usos industriales, y puede representar la solución a ciertos problemas de salud pública en muchos países, pero muchos de nosotros lo conocemos tan solo por los asados al aire libre con amigos y familiares. Existen otros usos que se le pueden dar a un biocarbón, sea que provenga de leña o de residuos agroindustriales. El biocarbón es un sustituto muy ventajoso de la biomasa en la cocina, ya que no emite humo. Con esta sustitución, se pueden reducir drásticamente las afecciones respiratorias de las personas que cocinan mediante la combustión de biomasa. Esto representa un tema importante de salud pública para una parte importante de la población humana mundial, e incluye a muchas personas en nuestro territorio.

Al anterior uso, se agrega el de mejorador de suelos, que cada día tiene más adeptos y cobra mayor interés en todo el mundo. El potencial del biocarbón como mejorador de suelos se redescubrió a partir de esfuerzos investigativos liderados por el maestro Wim Sombroek (6), padre de toda una corriente en la ciencia de suelos. En pocas palabras, Sombroek contribuyó a redescubrir que el biocarbón en el suelo actúa de manera casi milagrosa, haciendo que los suelos lleguen a multiplicar su productividad varias veces, y reduciendo la necesidad de insumos agrícolas, y por tanto los costos de producción (7, 8). El biocarbón en los suelos, por lo que se sabe, perdura por siglos o milenios, con efectos positivos esencialmente permanentes (I, 2). La evidencia está en suelos intervenidos por poblaciones humanas desaparecidas hace cientos o miles de años, suelos que conservan hoy características muy superiores a las de los suelos originales circundantes, no intervenidos. Las implicaciones de estos hechos son amplias y profundas, y son de gran interés investigativo en la actualidad. 


\section{Tendencias actuales}

El interés actual por la bioenergía para la sustitución de fuentes fósiles ha dado como resultado toda una variedad de investigaciones en opciones para procesar biomasa y obtener de ella productos químicos o energéticos. El interés se ha centrado en la obtención de energía, donde la combustión directa es el primero y más obvio recurso, pero su aplicabilidad es limitada por la distancia de transporte. Gran parte del esfuerzo alterno se ha dirigido a procesos pirolíticos, en los que el material es sometido a temperaturas elevadas en ausencia de aire, evitando la combustión, para que reaccione y dé los productos de interés.

Los procesos pirolíticos van desde la gasificación, a mayores temperaturas $\left(T>600^{\circ} \mathrm{C}\right)$, pasando por la pirolisis a temperaturas intermedias (en torno a los $500^{\circ} \mathrm{C}$ ), hasta la carbonización (entre $250^{\circ} \mathrm{C}$ y $500^{\circ} \mathrm{C}$ ). La gasificación produce gases, los cuales usualmente son utilizados inmediatamente para su combustión, sea en llamas o en motores de combustión interna. La pirólisis se enfoca principalmente en la obtención de productos líquidos, que pueden ser conservados para su posterior uso, sea en un proceso químico o como fuentes de energía. En ambos casos, suele producirse una cantidad significativa de carbón (en torno al 10\% de la masa original). La carbonización, que se realiza a menores temperaturas, tiene como producto principal productos carbonosos sólidos, y su rendimiento suele ser cercano al 30\% de la masa original.

Durante la carbonización de la biomasa suelen producirse gases que se condensan a temperatura ambiente, produciendo un aerosol que llamamos humo. El humo es una suspensión de diminutas gotas y partículas en el aire. La mezcla de sustancias que hay en el humo se puede utilizar para una variedad de propósitos, desde la obtención de energía hasta la aplicación a cultivos como fertilizante o plaguicida, pasando por algunos usos medicinales. Es similar al aceite de pirólisis, que se obtiene a temperaturas superiores con mucho mayores rendimientos. Para el caso de la producción industrial de biocarbón, probablemente el uso energético de estos aceites sea el más factible y práctico.

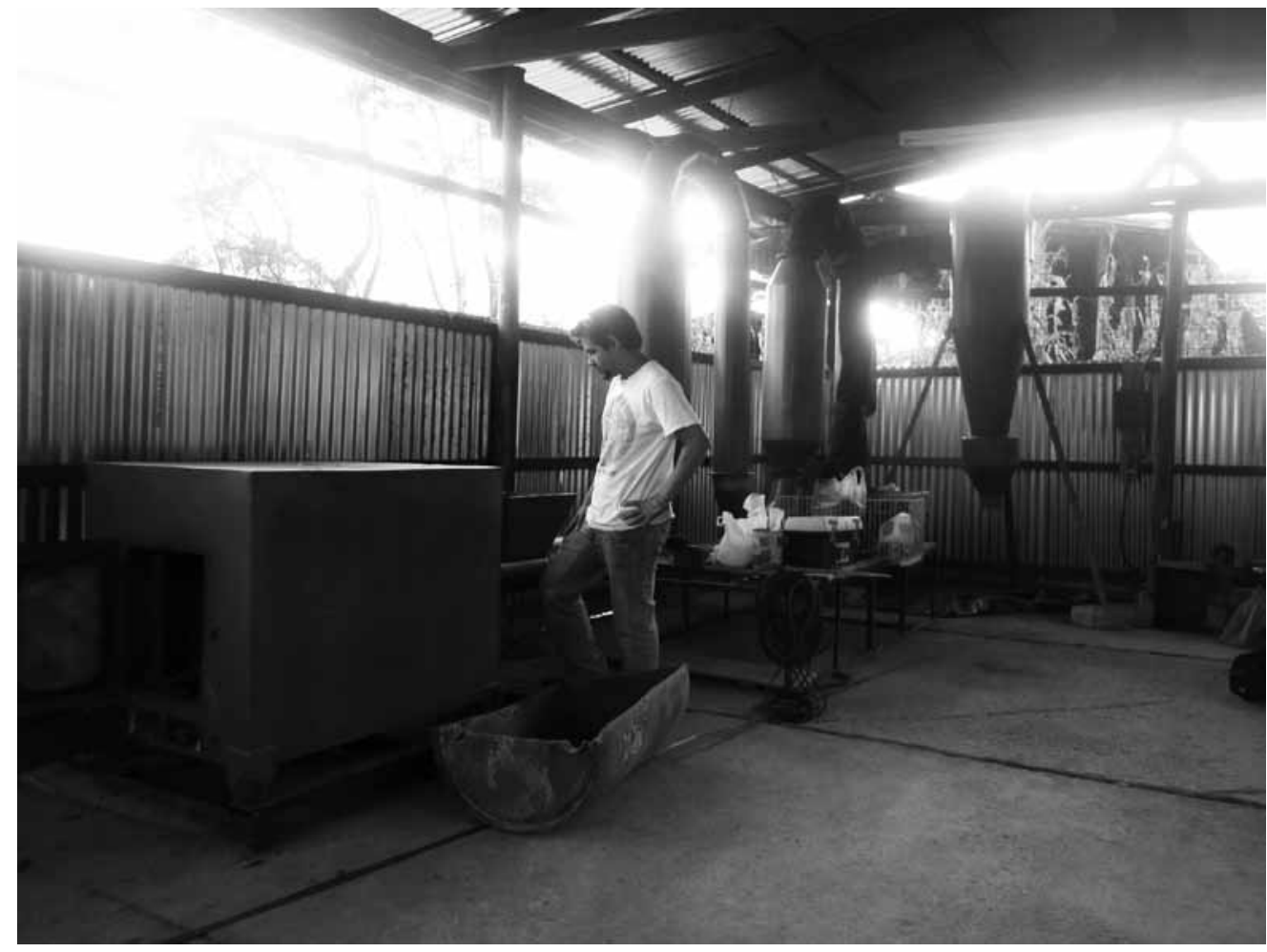

Secador de biomasa. Utiliza una corriente de aire calentado por medio de la combustión de leña o carbón. Puede procesar más de $100 \mathrm{~kg}$ por hora. 


\section{Los proyectos de carbonización de biomasa del TEC}

En el TEC ha existido por muchos años interés por las energías renovables. Este interés llevó en el 2009 a la conformación del programa de energías limpias (PELTEC), bajo el liderazgo del Ing. Carlos Roldán. Este programa procura agrupar a quienes investigamos en temas de energías renovables, a fin de generar sinergias. En el marco del PELTEC, en el 20 I I se inició un proyecto dirigido a la evaluación de la carbonización de residuos agrícolas y agroindustriales de la industria del café. Este proyecto nace del interés del autor por implantar localmente procesos y tecnologías que ayuden a convertir problemas, como los residuos agroindustriales difíciles, en fuentes de riqueza, obteniendo así múltiple ganancia y facilitando una pervivencia de los seres humanos más ambientalmente coherente y digna.

El primer fin del proyecto de carbonización en seco, iniciado en el 201 I, es evaluar los residuos biomásicos de mayor interés para la empresa Coopetarrazú, como fuentes de biocarbón, y evaluar la utilización de este en la planta, con propósitos energéticos, y en el campo, como mejorador de suelos. Otro de los fines es dejar una capacidad instalada en el TEC para evaluar la carbonización de residuos vegetales de cualquier origen, a fin de ofrecer este servicio a empresas interesadas en el aprovechamiento de sus residuos biomásicos mediante la carbonización. Igualmente podrá ser utilizada esa capacidad instalada para investigar otros casos, y en la implementación de una pequeña empresa productiva, en la que participen estudiantes de la institución, por ejemplo. Los residuos vegetales del campus podrán ser una buena materia prima.

Con este proyecto se logra, además, concretar el gran interés del TEC en establecer alianzas de cooperación universidad-empresa que tengan impacto, y en particular en el caso de Coopetarrazú, la cooperación ha sido sumamente productiva y eficaz.

El equipo adquirido para el proyecto comprende un molino, un secador, un briqueteador y un horno de carbonización con cerca de $3 \mathrm{~m}^{3}$ de capacidad. El molino permite moler el material a carbonizar, para que lo pueda manejar el briqueteador. El briqueteador forma briquetas de biomasa a partir del polvo que produce el molino; las briquetas se carbonizan posteriormente en el horno para producir briquetas de biocarbón. El secador permite llevar el polvo biomásico al grado de humedad que el briquetador requiere. La instalación del equipo en la planta de Coopetarrazú está casi concluida, y ya dieron inicio las pruebas iniciales para establecer las condiciones de producción del carbón que se necesitará para las subsecuentes pruebas. Se prevén pruebas en la aplicación energética del biocarbón en planta, y en la aplicación de mejoramiento de suelos.

\section{Carbonización hidrotérmica}

El proyecto iniciado en el 2012 está enfocado a evaluar la carbonización hidrotérmica, que es un fenómeno reportado ya a inicios del siglo pasado (9), como opción para el aprovechamiento de residuos con altos contenidos de agua. La carbonización hidrotérmica consiste en someter el material a temperaturas altas, sumergido en agua, sin permitir que haya ebullición. Se ha observado que por este tipo de reacción es posible carbonizar materiales lignocelulósicos sólidos, pero también polisacáridos disueltos en agua, obteniendo materiales carbonosos nanoestructurados como productos ( I0).

La particularidad de que requiere un medio acuoso resulta de gran ventaja para su aplicación a residuos que, justamente, tienen alto contenido de agua, y que requerirían de varios pasos de secado para poder ser carbonizados en seco. Es decir que los residuos se pueden carbonizar sin secarlos antes.

Este proyecto está enfocado en las aguamieles del beneficiado del café, que constituyen un problema muy significativo, y representan costos importantes de manejo. Evaluar la carbonización de la broza, con cerca de $90 \%$ de agua, es el siguiente paso lógico, una vez evaluadas las aguamieles.

En colaboración con la empresa Oventec, estamos en el proceso de diseñar y construir un carbonizador hidrotérmico de escala intermedia (del orden de I L/min). Una vez funcione apropiadamente el carbonizador hidrotérmico, y evaluadas las aguamieles, se podrán evaluar otros residuos con altos contenidos de agua. Igualmente se podrán ensayar diferentes condiciones de reacción para obtener variantes en los productos carbonosos, que podrán tener algunas características especiales, algunas de las cuales podrán ser examinadas por el grupo de nanotecnología delTEC. 


\section{Bibliografía}

I.- Glaser, B. (2007). Prehistorically modified soils of central Amazonia: a model for sustainable agriculture in the twenty-first century. Philosophic Transactions of the Royal Society B 362 (1478): I87-196.

2.- E.G. Neves, R.N. Bartone, J.B. Petersen \& M.J. Heckenberger (200I). The timing of Terra Preta formation in the central Amazon: new data from three sites in the central Amazon. p. 10.

3.- Erickson, Clark, (2003) Historical Ecology and Future Explorations En Lehmann, J.; Kern, D.C.; Glaser, B.; Woods, W.I. (Eds.) Amazonian Dark Earths: origin, properties, and management. Capítulo 23 (p. 484-ss).

4.- Ramírez. F. \& Roldán, C. (2007) Encuesta de oferta y consumo energético nacional a partir de la biomasa en Costa Rica, 2006. San José: MINAE.

5.- RECOPE (2008) Boletín (Boletín 27 del 18 de abril del 2008) San José: RECOPE.
6.- Lehmann, J. (2006). Black is the new green, Nature 442: 624-626.

7.- Laird, D. (2008) The charcoal vision: A win-win-win scenario for simultaneously producing bioenergy, permanently sequestering carbon, while improving soil and water quality. Agronomy Journal I00: |78-181.

8.- Lehmann, J., da Silva Jr., J.P., Steiner, C., Nehls, T., Zech,W. \& Glaser, B.: (2003). Nutrient availability and leaching in an archaeological Anthrosol and a Ferralsol of the Central Amazon basin: fertilizer, manure and charcoal amendments, Plant and Soil 249: 343-357.

9.- J. A. Libra, K. S. Ro, C. Kammann, A. Funke, N. D Berge, Y. Neubauer, M. M. Titirici, C. Fühner, O. Bens, J. Kern \& K. H. Emmerich (20I I). Hydrothermal Carbonization of Biomass Residuals: a Comparative Review of the Chemistry, Processes and Applications of Wet and Dry Pyrolysis, Biofuels, 2(I): 89

10.- N. Baccile, M.Antonietti, M. M.Titirici (20 I0) AlbuminDirected One-Step Synthesis of Nitrogen-Doped Hydrothermal Carbon Nanostructures from Glucose, ChemSusChem, 3(2): 122. 